\title{
Review and current state of radiation therapy for locally advanced pancreatic adenocarcinoma
}

\author{
Adriana Blakaj ${ }^{1}$, Stacey M. Stein ${ }^{2}$, Sajid A. Khan ${ }^{3}$, Kimberly L. Johung' \\ ${ }^{1}$ Department of Therapeutic Radiology, ${ }^{2}$ Department of Medicine, Section of Medical Oncology, ${ }^{3}$ Department of Surgery, Section of Surgical \\ Oncology, Yale University School of Medicine, New Haven, CT, USA \\ Contributions: (I) Conception and design: KL Johung; (II) Administrative support: KL Johung; (III) Provision of study materials or patients: None; \\ (IV) Collection and assembly of data: None; (V) Data analysis and interpretation: None; (VI) Manuscript writing: All authors; (VII) Final approval of \\ manuscript: All authors. \\ Correspondence to: Kimberly L. Johung, MD, PhD. Department of Therapeutic Radiology, Yale University School of Medicine, P.O. Box 208040, \\ New Haven, CT 06520-8040, USA. Email: kimberly.johung@yale.edu.
}

\begin{abstract}
Pancreatic cancer is characterized by a high rate of metastatic spread and overall poor prognosis. Yet $30 \%$ of patients have progressive local disease at the time of death, and local progression can cause significant morbidity. Approximately 30-40\% of patients present with locally advanced pancreatic cancer (LAPC) that is not surgically resectable, and the optimal treatment for these patients continues to evolve. The role of radiation in the management of LAPC is an area of controversy, and the recent LAP07 randomized trial reported no survival benefit of radiation following gemcitabine plus or minus erlotinib. However, the efficacy of modern systemic regimens has improved since the design of the LAP07 study, and radiation therapy may be of greater benefit in the context of more effective systemic therapy. Advances in radiation delivery including the increasing use of stereotactic body radiation therapy (SBRT) have the potential to improve outcomes through dose escalation and better treatment tolerability. In addition, the combination of radiation therapy and immune therapy is an area of promising research. These advances suggest that radiation therapy will continue to play an integral role in the management of LAPC.
\end{abstract}

Keywords: Locally advanced pancreatic cancer (LAPC); radiation

Submitted Feb 08, 2018. Accepted for publication Mar 22, 2018.

doi: 10.21037/jgo.2018.03.07

View this article at: http://dx.doi.org/10.21037/jgo.2018.03.07

\section{Background}

Despite advances in the treatment of pancreatic cancer, prognosis remains extremely poor with 5 -year survival of only $8 \%$ (1). Pancreatic cancer is currently the fourth leading cause of cancer-related death in the United States and is predicted to surpass breast, prostate, and colorectal cancers to become the second most common cause of cancer mortality by $2030(2,3)$. Patients with localized disease are considered resectable, borderline resectable, or locally advanced. While surgical resection offers the only potential for cure, only $15 \%$ of patients present with disease that is resectable upfront, and in these patients the 5-year survival is at best $20-25 \%(4,5)$. Patients with borderline resectable disease account for another $5-10 \%$ of cases, and neoadjuvant therapy is often utilized prior to proceeding with a pancreatectomy if there is no disease progression. Approximately $30-40 \%$ of patients have locally advanced unresectable pancreatic cancer (LAPC) in which tumor involvement of neighboring blood vessels prohibits surgery, and this class of tumor will be the focus of this review.

The National Comprehensive Cancer Network (NCCN) guidelines define unresectable tumors as tumors of the pancreatic head or uncinate process meeting any of the following criteria: (I) solid tumor contact with the superior mesenteric artery (SMA) >180 degrees; (II) solid tumor 
contact with the celiac artery (CA) $>180$ degrees; (III) solid tumor contact with the first jejunal branch of the SMA; (IV) tumor involvement or occlusion of the superior mesenteric vein (SMV) or portal vein (PV) with SMV/ $\mathrm{PV}$ reconstruction not feasible; (V) tumor contact with the most proximal draining jejunal branch into the SMV; or tumors of the pancreatic body or tail meeting one or more of the following criteria: (I) solid tumor contact with $>180$ degrees of the SMA or CA; (II) tumor contact with the CA and aorta; (III) unreconstructible SMV/PV due to tumor involvement or occlusion (6). Surgical resection of the primary tumor is rarely feasible in these patients, even after systemic therapy and/or radiation therapy. Effective treatment of LAPC is challenging, partly because of a high rate of distant metastatic progression, reaching 30-50\% within 3 months of diagnosis $(7,8)$. Thus, initial systemic therapy is important to address occult distant disease.

However, local tumor control is also critical. An autopsy study evaluating patterns of failure demonstrated that $30 \%$ of patients die with locally progressive disease (9). Local progression can be a significant cause of morbidity with resultant pain from celiac nerve plexus involvement, gastrointestinal bleeding from local vascular invasion, gastric outlet obstruction from mass effect of proximal tumors, and jaundice from obstruction of the distal biliary tree. Strategies to identify the subset of patients destined for local disease progression are emerging. Intact expression of the tumor suppressor SMAD4 has been shown to correlate with an increased likelihood of local disease progression, whereas loss of SMAD4 expression predicts for metastatic progression (9). Thus, while molecular profiling may increasingly guide treatment decisions in the future, current practice guidelines from the American Society of Clinical Oncology (ASCO) recommend initial combination systemic chemotherapy for 6 months and consideration of radiation therapy for those patients with local disease progression or stable disease but unacceptable toxicities after initial chemotherapy (10).

The role of radiation in the treatment of LAPC continues to evolve. The publication of the LAP07 trial called into question the benefit of radiation in the management of LAPC, as there was no survival benefit to chemoradiation compared to chemotherapy alone in patients without progression after 4 months of gemcitabine with or without erlotinib (11). Despite these results, advances both in systemic therapy regimens and radiation delivery likely impact the effect of radiation on outcomes. This review summarizes the available literature and novel prospects for the use of radiation therapy in the treatment of LAPC.

\section{Chemoradiation compared to chemotherapy alone}

Randomized trials comparing chemoradiation to systemic therapy alone have demonstrated conflicting results, leading to controversy regarding whether there is a benefit to employing radiation in the treatment of LAPC. Three early randomized studies compared chemotherapy to chemoradiation and maintenance chemotherapy, with differing results. A Gastrointestinal Tumor Study Group (GITSG) trial published in 1988 compared streptozocin, mitomycin, and 5-fluorouracil (SMF) chemotherapy with chemoradiation (54 Gy with concurrent 5-FU followed by SMF), and demonstrated an improvement in survival with chemoradiation (median survival of $10.5 \mathrm{vs} .8$ months with systemic therapy, $\mathrm{P}<0.02)(12)$. In contrast, the Eastern Cooperative Oncology Group (ECOG) trial randomized patients to bolus $5-\mathrm{FU}$ or radiation to $40 \mathrm{~Gy}$ with concurrent and maintenance $5-\mathrm{FU}$, showing equivalent survival in the chemotherapy alone and chemoradiation arms (8.2 vs. 8.3 months, respectively) (13). Finally, a Canadian study compared multi-drug chemotherapy (5-FU and methyl-CCNU) with chemoradiation to 46 Gy and also showed no difference in median survival between the two groups (7.3 vs. 7.8 months, respectively) (14). The lack of survival benefit seen with chemoradiation may in part be attributed to the mixed population of patients included in the ECOG study (unresectable disease as well as recurrent or residual disease after resection), and the lower radiation doses prescribed in both the ECOG and Canadian studies. But more importantly, outcomes from these studies are not applicable to current practice, as outdated chemotherapy regimens and radiation techniques were utilized.

More contemporary studies conducted by the Federation Francophone de Cancerologie Digestive (FFCD) and the Societe Francophone de Radiotherapie Oncologique (SFRO), as well as the ECOG again showed conflicting results. The 2000-01 FFCD/SFRO trial randomized 119 patients with locally advanced disease to gemcitabine alone, or an aggressive regimen of chemoradiation to 60 Gy with concurrent continuous infusion 5-FU and cisplatin, followed by maintenance gemcitabine. Conformal radiation was employed, but fields were larger than current practice as regional nodes were electively included. Grades 3-4 treatment-related toxicities were increased 
on the combined-modality arm leading to treatment breaks. Ultimately accrual was terminated early, with results showing inferiority of chemoradiation compared to gemcitabine alone (median survival 8.6 vs. 13 months, $\mathrm{P}=0.03$ ) (15). Because the chemotherapy differed between the treatment arms and the chemoradiation regimen entailed dose escalation to 60 Gy with multi-agent chemotherapy, it is difficult to draw conclusions regarding the potential benefit of chemoradiation. The contemporaneous ECOG E4201 trial compared chemoradiation to 50.4 Gy with concurrent gemcitabine to gemcitabine alone. Accrual was slow and terminated early at 74 patients. A small but significantly improved overall survival was demonstrated with the addition of radiation therapy to gemcitabine (11.1 vs. 9.2 months, $\mathrm{P}=0.017$ ). Additionally, the incidence of grade 3 or 4 toxicities were similar in the two arms, though grade 4 and 5 toxicities were increased with chemoradiation (16). The survival benefit with combined modality therapy, though modest, established a role for chemoradiation in the current management of LAPC.

The potential benefit of chemoradiation is supported by a recent Surveillance, Epidemiology, and End Results Program (SEER) analysis and meta-analysis. A total of 4,460 patients with LAPC were identified in the SEER database from 2004-2011, of whom 59\% received radiation. Survival was prolonged with the use of radiation ( $43 \%$ vs. $29 \%$ at one year, $\mathrm{P}<0.001)$, and the survival benefit persisted in multivariate analyses and propensity score-matched cohorts (17). Additionally, a meta-analysis of five randomized studies comparing chemoradiation to chemotherapy in patients with LAPC showed a trend, though nonsignificant, to a survival advantage with chemoradiation in the treatment of LAPC (18). Taken together, these findings establish a role for chemoradiation in the treatment paradigm for LAPC. While results are conflicting when upfront chemoradiation is compared to chemotherapy alone, the addition of radiation is likely of benefit in select patients.

\section{Chemoradiation as consolidation after chemotherapy}

The high rate of early distant progression indicates that many patients diagnosed with locally advanced disease harbor micrometastatic disease. Thus, the importance of upfront multi-agent systemic therapy is now reflected in consensus guidelines $(6,10)$. Retrospective and phase II studies support the selected use of chemoradiation in patients without early metastatic progression after several months of chemotherapy, suggesting that upfront systemic therapy not only addresses micrometastatic disease but also can be used to select patients that are more likely to derive benefit from local control with chemoradiation.

A retrospective analysis of patients enrolled in phase II and III Groupe Coopérateur Multidisciplinaire en Oncologie (GERCOR) studies compared outcomes with chemoradiation to continued chemotherapy after a minimum of 3 months of 5-FU or gemcitabine-based chemotherapy. Approximately $30 \%$ of patients developed metastatic disease after initial chemotherapy. Among those patients without progression, chemoradiation to 55 Gy with concurrent continuous infusion 5-FU improved survival compared to continued chemotherapy (median survival of 15.0 vs. 11.7 months, $\mathrm{P}=0.0009)$ (7). Similar outcomes were demonstrated in a single institution retrospective study at MD Anderson Cancer Center (MDACC), in which 300 patients received upfront chemoradiation (30 Gy with concurrent 5-FU, capecitabine, or gemcitabine) or chemoradiation after a median of 2.5 months of induction chemotherapy. Selected use of chemoradiation after initial chemotherapy was associated with improved survival (median of 11.9 months) compared to upfront chemoradiation (median survival 8.5 months, $\mathrm{P}<0.001$ ) (19). Multiple phase II studies have shown similar promising survival outcomes, with median survival ranging from 12.6 to 19.2 months when chemoradiation is employed after several months of chemotherapy (20-23). In addition, a recent analysis of the National Cancer Database (NCDB) included over 8,500 patients diagnosed with LAPC from 2004-2014 and multivariate analysis showed improved survival with chemoradiation compared to chemotherapy (median survival of $13.5 v s .10 .6$ months, respectively). Moreover, receipt of induction chemotherapy before chemoradiation was an independent predictor of improved survival (24). Thus, by excluding patients with early distant progression and addressing micrometastatic disease, induction chemotherapy may select patients with LAPC for optimal benefit from consolidative chemoradiation, and this treatment approach is supported by the current ASCO guidelines (10).

The promising results of these retrospective and phase II studies led to the phase III randomized LAP07 trial. This study included nearly 450 patients that were randomized to receive 4 months of gemcitabine with or without erlotinib. Those patients without disease progression were then randomized to chemoradiation (54 Gy with capecitabine) or continued chemotherapy for 2 months. Despite the survival 
advantages seen with chemoradiation in the previous studies, chemoradiation did not prolong survival over continued chemotherapy in this trial (median survival of 15.2 months with chemoradiation compared to 16.5 months with chemotherapy, $\mathrm{P}=0.83)$. However, chemoradiation was associated with improved local control (68\% vs. 54\%) and prolonged time to second line treatment (6.1 months compared to 3.7 months, $\mathrm{P}=0.02$ ) (11). Because lifespan is limited for patients with locally advanced pancreatic cancer (LAPC), the extended treatment-free interval is meaningful and likely improves quality of life. Moreover, while survival outcomes with the addition of chemoradiation did not replicate the results seen in the prior early phase and retrospective studies, radiation continues to be evaluated in the context of improved systemic regimens.

\section{Radiation in the era of FOLFIRINOX and gemcitabine/nab-paclitaxel}

Significant advances in systemic therapy have improved outcomes for patients with advanced pancreatic cancer and likely impact the role of radiation as well. The phase III PRODIGE4/ACCORD11 study compared FOLFIRINOX (5-FU, leucovorin, oxaliplatin, and irinotecan) with gemcitabine in patients with metastatic pancreatic cancer and found that FOLFIRINOX nearly doubled median overall survival compared to gemcitabine (11.1 vs. 6.8 months, $\mathrm{P}<0.0001$ ) (25). Additionally, the Metastatic Pancreatic Adenocarcinoma Clinical Trial (MPACT trial) demonstrated superiority of gemcitabine and nab-paclitaxel compared to gemcitabine alone in the metastatic setting, with median overall survival of $8.5 \mathrm{vs}$. 6.7 months, respectively (26). Yet, the gemcitabine and nabpaclitaxel regimen has not been compared prospectively with FOLFIRINOX. However improved progression free survival and tolerability of gemcitabine and nab-paclitaxel compared to FOLFIRINOX was demonstrated in a small single-institution retrospective series from Japan (27).

Given the efficacy demonstrated in metastatic pancreatic cancer, FOLFIRINOX and gemcitabine/nab-paclitaxel are now used in the treatment of LAPC. The phase II LAPACT study evaluated the efficacy and safety of gemcitabine/nab-paclitaxel for LAPC. Patients received 6 cycles of gemcitabine/nab-paclitaxel followed by continued chemotherapy, chemoradiation, or surgery if feasible. Results were promising with a median time to treatment failure of 8.6 months and median progression-free survival of 10.2 months (28). Similarly, multiple retrospective and early phase prospective studies have examined both the efficacy and tolerability of FOLFIRINOX as first-line therapy in LAPC, demonstrating rates of progression free survival and overall survival that compare favorably to historical studies (29-40). A multicenter study from France reported outcomes for the largest cohort of patients with LAPC treated with FOLFIRINOX. After a median of 5 cycles of chemotherapy, $75 \%$ of patients went on to receive consolidative therapy with either radiation (70\%) and/or surgery (36\%). Median progression survival was 13 months and median overall survival was 22 months (33). Improved response rates with modern systemic regimens may increase the number of patients deemed appropriate for resection despite an initial presentation of locally advanced disease $(31,32,37)$. A small retrospective study of patients with LAPC treated with FOLFIRINOX followed by chemoradiation at Massachusetts General Hospital reported R0 resections achieved in 5 of 22 patients (32). Finally, a recent meta-analysis of mostly retrospective cohort studies evaluated outcomes with first-line FOLFIRINOX for LAPC. Median progression free survival was 15 months, and median overall survival was 24.2 months, which is significantly prolonged compared to historical controls including patients in the LAP07 trial, where the median survival in patients who received induction chemotherapy and chemoradiation was 15.2 months $(11,41)$. Of the 13 studies and approximately 700 patients included, only a fraction of these studies reported on the use of radiation. In the 8 studies that reported details of radiation therapy, $57 \%$ of patients received either conventional radiation, chemoradiation, or stereotactic body radiation therapy (SBRT). The proportion of patients receiving radiation did not correlate with survival, however survival outcomes of the subset of patients who received radiation were not reported (41).

The prolonged survival reported with the use of FOLFIRINOX and gemcitabine/nab-paclitaxel in LAPC suggests improved control of micrometastatic disease and distant progression, and it follows that the addition of local therapy with radiation is more likely to favorably impact outcomes in this setting.

\section{Advances in radiation techniques}

\section{Intensity modulated radiation therapy (IMRT)}

Technological advances in radiation delivery, which allow for reduced treatment-related toxicities and escalation of 

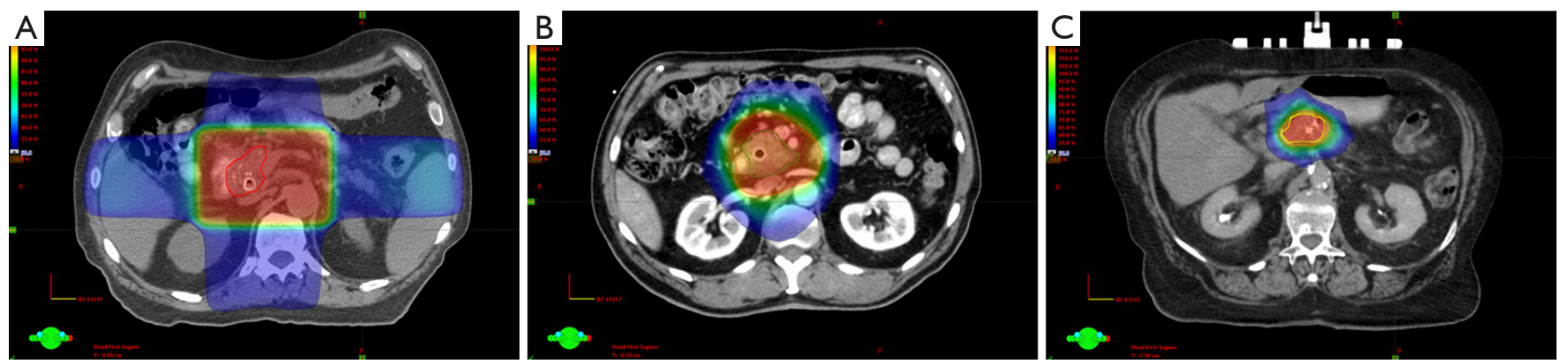

Figure 1 Representative axial images of a 3D conformal radiation plan to 50.4 Gy in 28 fractions (A), an intensity modulated radiation therapy (IMRT) plan to 50.4 Gy in 28 fractions (B), and a stereotactic body radiation therapy (SBRT) plan to 33 Gy in 5 fractions (C) for patients with locally advanced pancreatic cancer. The $50 \%$ isodose cloud is shown for each plan.

dose delivered, will also impact the efficacy of radiation in the management of LAPC. In comparison to 3D conformal radiation, in which multiple beams are shaped to match the tumor target, IMRT better conforms the dose delivered to the treatment volume by modulating the intensity of the radiation beam over its aperture, reducing toxicities of treatment and allowing for dose escalation by limiting dose to neighboring normal tissue. The advantage of using IMRT in the treatment of pancreatic cancer was demonstrated in a retrospective study of over 200 patients receiving chemoradiation for LAPC. This study showed decreased grade 2 or higher gastrointestinal (GI) toxicity with IMRT compared to 3D conformal techniques (34\% vs. $16 \%$, respectively). Interestingly, the median radiation dose was significantly higher in the IMRT treatment plans (56 vs. 50.4 Gy), thus suggesting that IMRT, by reducing GI toxicity, may allow for dose escalation to the tumor (42).

An analysis of the NCDB evaluating patterns of care for LAPC demonstrated an overall decline in the use of radiation, but an increase in usage of IMRT from $27-72 \%$ among those patients receiving radiation from 2003 through 2011. Interestingly, survival was improved compared to chemotherapy alone when chemoradiation was delivered with IMRT, but not 3D conformal radiation (43). These results suggest that the use of IMRT improves the therapeutic index of radiation therapy, and radiation may improve outcomes in LAPC when advanced techniques are utilized.

\section{SBRT}

SBRT delivers ablative doses of radiation to the tumor volume with minimal margin and is increasingly being used in the treatment of pancreatic cancer. An example of a SBRT plan compared to an IMRT plan and a 3D conformal radiation plan for LAPC is shown in Figure 1, demonstrating the more conformal dose distribution with IMRT and SBRT, and reduced treatment margins with SBRT. Multiple phase I-II or retrospective single institution studies have evaluated the use of SBRT for LAPC. Early studies from Stanford University explored single fraction treatment alone, as a boost after conventionally fractionated radiation, or in combination with sequential gemcitabine. While high rates of local control were reported at 1 year (94-100\%), significant grade 2 or higher late GI toxicities (20-44\%) were observed, including duodenal ulcers, and less commonly stenosis or perforation (44-47). Subsequent studies utilized a multi-fraction approach [24-36 Gy in 3 fractions (48-50), 25-30 Gy in 5 fractions with a simultaneous integrated boost of $35-40$ Gy to areas of vessel abutment $(37,51)$, or 36-45 Gy in 6 fractions $(52,53)]$ and maintained effective local control (75-90\% at 1 year) with reduced GI toxicities. These studies include a prospective multi-institutional phase II study, which evaluated gemcitabine followed by fractionated SBRT (54). Patients received up to 3 weeks of gemcitabine $\left(1,000 \mathrm{mg} / \mathrm{m}^{2}\right)$ followed one week later by SBRT to $33 \mathrm{~Gy}$ in 5 fractions delivered over 1 to 2 weeks, and gemcitabine until disease progression or toxicity. The primary endpoint was late grade 2 or higher GI toxicities compared to historical controls. When compared to prior studies using single-fraction SBRT, GI toxicities were reduced to $2 \%$ grade 2 or higher acute toxicity, and $11 \%$ grade 2 or higher late toxicity. The median overall survival was 13.9 months. Local control at 1 year was $78 \%$, which compares favorably to historical controls treated with conventionally fractionated radiation, such as the LAP07 trial in which local control with chemoradiation was $68 \%$ 
$(11,54)$. In addition, quality of life data collected from patients enrolled on this study demonstrated no change in overall quality of life at one and four-month follow-up intervals after SBRT, suggesting that treatment was well tolerated (55).

Evidence to date supports the continued evaluation of SBRT in the management of LAPC. A pooled analysis of 19 studies and over 1,000 patients with LAPC treated with SBRT reported promising outcomes, with acceptable rates of toxicity, locoregional control at one year of $72.3 \%$, and median overall survival of 17 months (56). While prospective studies to date have not compared SBRT to conventionally fractionated radiation, independent analyses of the NCDB showed improved survival with SBRT compared to conventionally fractionated radiation $(57,58)$.

The advantages of SBRT include the fact that the biologic effect of larger radiation dose per fraction may provide a therapeutic benefit with improved local control compared to conventionally dosed radiation. Also, the shorter treatment course can be better integrated with systemic therapy, which can result in less time off effective multi-agent chemotherapy. Thus, SBRT may improve outcomes in the context of modern systemic therapy regimens, and a phase III study is underway to evaluate FOLFIRINOX with or without SBRT for LAPC (ClinicalTrials.gov identifier NCT01926197).

\section{Dose escalation with IMRT and hypofractionation}

The ability to deliver definitive radiation doses to pancreatic tumors is limited by the radiation tolerance of the neighboring small bowel and stomach. While SBRT is one approach to deliver a higher biological effective dose (BED), hypofractionated radiation is another approach in which the total radiation course is shortened, and daily fractions are larger compared to conventionally fractionated radiation, but unlike SBRT, daily fractions over the course of weeks are maintained to take advantage of normal tissue repair between fractions. SBRT techniques are necessary to achieve dose escalation with hypofractionation, including methods to account for the respiratory motion of tumors with 4-dimensional planning CT scans, respiratory gating to treat only in certain phases of the respiratory cycle, abdominal compression to minimize respiratory motion during treatment, as well as placement of fiducials in tumors to guide daily treatment alignment (59).

A series of 200 patients with LAPC treated at MDACC included 47 patients with tumors at least $1 \mathrm{~cm}$ away from bowel. In these patients, IMRT was used to escalate dose to the tumor with a hypofractionated regimen (most commonly 63-70 Gy in 28 fractions or 67.5 Gy in 15 fractions). Regions of bowel abutting the tumor were underdosed if necessary to meet normal tissue constraints. Overall survival was improved when a BED greater than 70 Gy was delivered. Interestingly the degree of tumor coverage did not correlate with outcomes, suggesting that escalation of dose to even a portion of the gross tumor volume is of benefit (60).

Increasing the prescribed dose of radiation may improve the rate of resectability in patients with locally advanced disease. A phase I/II trial of 50 patients with unresectable pancreatic cancer evaluated the efficacy and tolerability of dose-escalated IMRT using a breath hold technique with concurrent gemcitabine. Radiation dose was escalated from a standard dose of $50 \mathrm{~Gy}$ in 25 fractions to $60 \mathrm{~Gy}$ in 25 fractions, with a final recommended dose of 55 Gy at which the probability of a dose limiting toxicity was 0.24 . Median survival was 14.8 months, and a large fraction of patients converted to resectable disease with $\mathrm{R} 0$ resections achieved in $20 \%$ of patients at a median of 7.5 months from initiation of treatment (61). Dose-painting techniques in which regions of blood vessel involvement receive a higher dose than the remainder of the tumor have also been evaluated. In a recent study, the tumor volume and regional nodes received a standard dose of 50.4 Gy in 28 fractions of $1.8 \mathrm{~Gy}$ while the region around involved vessels was simultaneously boosted to 58.8 Gy in 2.1 Gy fractions. With this neoadjuvant chemoradiation regimen, $37 \%$ of initially unresectable patients were converted to surgically resectable. Acute grade 3 or greater nausea, fatigue, and diarrhea rates were $12 \%, 10 \%$, and $6 \%$, respectively. Late grade 3 or greater duodenal toxicity was $5 \%$ (62). Thus, dose escalation is feasible and tolerable if correctly executed and may improve tumor control and resectability for patients with LAPC.

Technological advances continue to improve the ability to deliver ablative doses of radiation to the tumor volume while protecting neighboring normal tissues. One such innovation is the use of a radiopaque hydrogel to space the head of the pancreas from the duodenum. A study in human cadavers demonstrated the feasibility of injecting the spacer between the duodenum and pancreas under EUS guidance, creating on average one centimeter of space. SBRT plans were then modeled on the pre- and post-hydrogel scans demonstrating a significant reduction in dose delivered to the duodenum in the presence of the spacer (63). If 
feasible in patients, this spacer has the potential to improve outcomes in LAPC by allowing for safe dose escalation.

\section{Immune response to radiation}

While immunotherapy has revolutionized the treatment of many tumor types, responses in patients with advanced pancreatic cancer have been disappointing $(64,65)$, likely in part due to the immunosuppressive pancreatic tumor microenvironment. Pancreatic tumors are characterized by a dense desmoplastic stroma and infiltration of predominantly immunosuppressive leukocytes including tumor-associated macrophages and regulatory $\mathrm{T}$ cells with rare effector T-cells, which together promote evasion of the immune system $(66,67)$. Radiotherapy has been shown to have immunomodulatory effects, which may influence the response to immunotherapy if used in conjunction. Radiation triggers the release of tumor antigens, recruits effector $\mathrm{T}$ cells through induction of chemokines, and increases expression of co-stimulatory molecules and death receptors on tumor cells, which enhances anti-tumor immunity. Radiation can also stimulate immunosuppressive leukocytes and induce expression of programmed deathligand 1 (PD-L1) (68-70). Potential synergy between radiation and immune checkpoint inhibitors to promote anti-tumor immunity is an area of active research (70). In pancreatic cancer, radiation has been shown to upregulate PD-L1 expression. Moreover, in mouse models of pancreatic cancer, PD-L1 blockade had no effect on tumor growth whereas radiation slowed tumor growth as expected. Interestingly the combination of radiation and anti-PD-L1 had a synergistic effect to inhibit tumor growth, and this response was dependent on infiltration of CD8+ $\mathrm{T}$ cells and was restricted to only larger fractions of radiation but not lower doses (71). Thus, the use of radiation in combination with immune checkpoint inhibitors may improve outcomes for patients with advanced pancreatic cancer both through local tumor responses as well as possible abscopal responses, and numerous clinical trials are ongoing to evaluate this theory.

\section{Conclusions}

Pancreatic cancer remains one of the leading causes of cancer-related mortality, and many patients present with locally advanced unresectable disease. The role of radiation in the management of these patients has historically been controversial, as existing randomized data has shown conflicting results. However, with advances in systemic therapy improving control of micrometastatic disease, advances in radiation delivery allowing for dose escalation, as well as possible synergy between radiation and immune therapy, there is great potential for radiation to improve future outcomes in LAPC.

\section{Acknowledgements}

None.

\section{Footnote}

Conflicts of Interest: The authors have no conflicts of interest to declare.

\section{References}

1. American Cancer Society. Cancer Facts and Figures 2018. Atlanta: American Cancer Society, 2018.

2. Siegel RL, Miller KD, Jemal A. Cancer Statistics, 2017. CA Cancer J Clin 2017;67:7-30.

3. Rahib L, Smith BD, Aizenberg R, et al. Projecting cancer incidence and deaths to 2030: the unexpected burden of thyroid, liver, and pancreas cancers in the United States. Cancer Res 2014;74:2913-21.

4. Neoptolemos JP, Palmer DH, Ghaneh P, et al. Comparison of adjuvant gemcitabine and capecitabine with gemcitabine monotherapy in patients with resected pancreatic cancer (ESPAC-4): a multicentre, open-label, randomised, phase 3 trial. Lancet 2017;389:1011-24.

5. Oettle H, Neuhaus $P$, Hochhaus A, et al. Adjuvant chemotherapy with gemcitabine and long-term outcomes among patients with resected pancreatic cancer: the CONKO-001 randomized trial. JAMA 2013;310:1473-81.

6. Tempero MA, Malafa MP, Al-Hawary M, et al. Pancreatic Adenocarcinoma, Version 2.2017, NCCN Clinical Practice Guidelines in Oncology. J Natl Compr Canc Netw 2017;15:1028-61.

7. Huguet F, Andre T, Hammel P, et al. Impact of chemoradiotherapy after disease control with chemotherapy in locally advanced pancreatic adenocarcinoma in GERCOR phase II and III studies. J Clin Oncol 2007;25:326-31.

8. Mukherjee S, Hurt CN, Bridgewater J, et al. Gemcitabinebased or capecitabine-based chemoradiotherapy for locally 
advanced pancreatic cancer (SCALOP): a multicentre, randomised, phase 2 trial. Lancet Oncol 2013;14:317-26.

9. Iacobuzio-Donahue CA, Fu B, Yachida S, et al. DPC4 gene status of the primary carcinoma correlates with patterns of failure in patients with pancreatic cancer. J Clin Oncol 2009;27:1806-13.

10. Balaban EP, Mangu PB, Khorana AA, et al. Locally Advanced, Unresectable Pancreatic Cancer: American Society of Clinical Oncology Clinical Practice Guideline. J Clin Oncol 2016;34:2654-68.

11. Hammel P, Huguet F, van Laethem JL, et al. Effect of Chemoradiotherapy vs Chemotherapy on Survival in Patients With Locally Advanced Pancreatic Cancer Controlled After 4 Months of Gemcitabine With or Without Erlotinib: The LAP07 Randomized Clinical Trial. JAMA 2016;315:1844-53.

12. Treatment of locally unresectable carcinoma of the pancreas: comparison of combined-modality therapy (chemotherapy plus radiotherapy) to chemotherapy alone. Gastrointestinal Tumor Study Group. J Natl Cancer Inst 1988;80:751-5.

13. Klaassen DJ, MacIntyre JM, Catton GE, et al. Treatment of locally unresectable cancer of the stomach and pancreas: a randomized comparison of 5 -fluorouracil alone with radiation plus concurrent and maintenance 5-fluorouracil-an Eastern Cooperative Oncology Group study. J Clin Oncol 1985;3:373-8.

14. Hazel JJ, Thirlwell MP, Huggins M, et al. Multi-drug chemotherapy with and without radiation for carcinoma of the stomach and pancreas: a prospective randomized trial. J Can Assoc Radiol 1981;32:164-5.

15. Chauffert B, Mornex F, Bonnetain F, et al. Phase III trial comparing intensive induction chemoradiotherapy (60 Gy, infusional 5-FU and intermittent cisplatin) followed by maintenance gemcitabine with gemcitabine alone for locally advanced unresectable pancreatic cancer. Definitive results of the 2000-01 FFCD/SFRO study. Ann Oncol 2008;19:1592-9.

16. Loehrer PJ Sr, Feng Y, Cardenes H, et al. Gemcitabine alone versus gemcitabine plus radiotherapy in patients with locally advanced pancreatic cancer: an Eastern Cooperative Oncology Group trial. J Clin Oncol 2011;29:4105-12.

17. Sajjad M, Batra S, Hoffe S, et al. Use of Radiation Therapy in Locally Advanced Pancreatic Cancer Improves Survival: A SEER Database Analysis. Am J Clin Oncol 2018;41:236-41.

18. Ambe C, Fulp W, Springett G, et al. A Meta-analysis of Randomized Clinical Trials of Chemoradiation Therapy in Locally Advanced Pancreatic Cancer. J Gastrointest
Cancer 2015;46:284-90.

19. Krishnan S, Rana V, Janjan NA, et al. Induction chemotherapy selects patients with locally advanced, unresectable pancreatic cancer for optimal benefit from consolidative chemoradiation therapy. Cancer 2007;110:47-55.

20. Moureau-Zabotto L, Phelip JM, Afchain P, et al. Concomitant administration of weekly oxaliplatin, fluorouracil continuous infusion, and radiotherapy after 2 months of gemcitabine and oxaliplatin induction in patients with locally advanced pancreatic cancer: a Groupe Coordinateur Multidisciplinaire en Oncologie phase II study. J Clin Oncol 2008;26:1080-5.

21. Schneider BJ, Ben-Josef E, McGinn CJ, et al. Capecitabine and radiation therapy preceded and followed by combination chemotherapy in advanced pancreatic cancer. Int J Radiat Oncol Biol Phys 2005;63:1325-30.

22. Crane CH, Varadhachary GR, Yordy JS, et al. Phase II trial of cetuximab, gemcitabine, and oxaliplatin followed by chemoradiation with cetuximab for locally advanced (T4) pancreatic adenocarcinoma: correlation of Smad4(Dpc4) immunostaining with pattern of disease progression. J Clin Oncol 2011;29:3037-43.

23. Ko AH, Quivey JM, Venook AP, et al. A phase II study of fixed-dose rate gemcitabine plus low-dose cisplatin followed by consolidative chemoradiation for locally advanced pancreatic cancer. Int J Radiat Oncol Biol Phys 2007;68:809-16.

24. Torgeson A, Lloyd S, Boothe D, et al. Multiagent induction chemotherapy followed by chemoradiation is associated with improved survival in locally advanced pancreatic cancer. Cancer 2017;123:3816-24.

25. Conroy T, Desseigne F, Ychou M, et al. FOLFIRINOX versus gemcitabine for metastatic pancreatic cancer. $\mathrm{N}$ Engl J Med 2011;364:1817-25.

26. Von Hoff DD, Ervin T, Arena FP, et al. Increased survival in pancreatic cancer with nab-paclitaxel plus gemcitabine. N Engl J Med 2013;369:1691-703.

27. Muranaka T, Kuwatani M, Komatsu Y, et al. Comparison of efficacy and toxicity of FOLFIRINOX and gemcitabine with nab-paclitaxel in unresectable pancreatic cancer. J Gastrointest Oncol 2017;8:566-71.

28. Hammel P, Lacy J, Portales F, et al. Phase II LAPACT trial of nab-paclitaxel (nab-P) plus gemcitabine $(\mathrm{G})$ for patients with locally advanced pancreatic cancer (LAPC). J Clin Oncol 2018;36:abstr 204.

29. Stein SM, James ES, Deng Y, et al. Final analysis of a phase II study of modified FOLFIRINOX in locally 
advanced and metastatic pancreatic cancer. Br J Cancer 2016;114:737-43.

30. Peddi PF, Lubner S, McWilliams R, et al. Multiinstitutional experience with FOLFIRINOX in pancreatic adenocarcinoma. JOP 2012;13:497-501.

31. Boone BA, Steve J, Krasinskas AM, et al. Outcomes with FOLFIRINOX for borderline resectable and locally unresectable pancreatic cancer. J Surg Oncol 2013;108:236-41.

32. Faris JE, Blaszkowsky LS, McDermott S, et al. FOLFIRINOX in locally advanced pancreatic cancer: the Massachusetts General Hospital Cancer Center experience. Oncologist 2013;18:543-8.

33. Marthey L, Sa-Cunha A, Blanc JF, et al. FOLFIRINOX for locally advanced pancreatic adenocarcinoma: results of an AGEO multicenter prospective observational cohort. Ann Surg Oncol 2015;22:295-301.

34. Gunturu KS, Yao X, Cong X, et al. FOLFIRINOX for locally advanced and metastatic pancreatic cancer: single institution retrospective review of efficacy and toxicity. Med Oncol 2013;30:361.

35. Sadot E, Doussot A, O'Reilly EM, et al. FOLFIRINOX Induction Therapy for Stage 3 Pancreatic Adenocarcinoma. Ann Surg Oncol 2015;22:3512-21.

36. Ferrone CR, Marchegiani G, Hong TS, et al. Radiological and surgical implications of neoadjuvant treatment with FOLFIRINOX for locally advanced and borderline resectable pancreatic cancer. Ann Surg 2015;261:12-7.

37. Mellon EA, Hoffe SE, Springett GM, et al. Long-term outcomes of induction chemotherapy and neoadjuvant stereotactic body radiotherapy for borderline resectable and locally advanced pancreatic adenocarcinoma. Acta Oncol 2015;54:979-85.

38. Moorcraft SY, Khan K, Peckitt C, et al. FOLFIRINOX for locally advanced or metastatic pancreatic ductal adenocarcinoma: the Royal Marsden experience. Clin Colorectal Cancer 2014;13:232-8.

39. Mahaseth H, Brutcher E, Kauh J, et al. Modified FOLFIRINOX regimen with improved safety and maintained efficacy in pancreatic adenocarcinoma. Pancreas 2013;42:1311-5.

40. Nanda RH, El-Rayes B, Maithel SK, et al. Neoadjuvant modified FOLFIRINOX and chemoradiation therapy for locally advanced pancreatic cancer improves resectability. J Surg Oncol 2015;111:1028-34.

41. Suker M, Beumer BR, Sadot E, et al. FOLFIRINOX for locally advanced pancreatic cancer: a systematic review and patient-level meta-analysis. Lancet Oncol 2016;17:801-10.
42. Prasad S, Cambridge L, Huguet F, et al. Intensity modulated radiation therapy reduces gastrointestinal toxicity in locally advanced pancreas cancer. Pract Radiat Oncol 2016;6:78-85.

43. Amini A, Jones BL, Stumpf P, et al. Patterns of Care for Locally Advanced Pancreatic Adenocarcinoma Using the National Cancer Database. Pancreas 2017;46:904-12.

44. Koong AC, Le QT, Ho A, et al. Phase I study of stereotactic radiosurgery in patients with locally advanced pancreatic cancer. Int J Radiat Oncol Biol Phys 2004;58:1017-21.

45. Koong AC, Christofferson E, Le QT, et al. Phase II study to assess the efficacy of conventionally fractionated radiotherapy followed by a stereotactic radiosurgery boost in patients with locally advanced pancreatic cancer. Int J Radiat Oncol Biol Phys 2005;63:320-3.

46. Schellenberg D, Goodman KA, Lee F, et al. Gemcitabine chemotherapy and single-fraction stereotactic body radiotherapy for locally advanced pancreatic cancer. Int J Radiat Oncol Biol Phys 2008;72:678-86.

47. Schellenberg D, Kim J, Christman-Skieller C, et al. Single-fraction stereotactic body radiation therapy and sequential gemcitabine for the treatment of locally advanced pancreatic cancer. Int J Radiat Oncol Biol Phys 2011;81:181-8.

48. Polistina F, Costantin G, Casamassima F, et al. Unresectable locally advanced pancreatic cancer: a multimodal treatment using neoadjuvant chemoradiotherapy (gemcitabine plus stereotactic radiosurgery) and subsequent surgical exploration. Ann Surg Oncol 2010;17:2092-101.

49. Mahadevan A, Jain S, Goldstein M, et al. Stereotactic body radiotherapy and gemcitabine for locally advanced pancreatic cancer. Int J Radiat Oncol Biol Phys 2010;78:735-42.

50. Mahadevan A, Miksad R, Goldstein M, et al. Induction gemcitabine and stereotactic body radiotherapy for locally advanced nonmetastatic pancreas cancer. Int J Radiat Oncol Biol Phys 2011;81:e615-22.

51. Chuong MD, Springett GM, Freilich JM, et al. Stereotactic body radiation therapy for locally advanced and borderline resectable pancreatic cancer is effective and well tolerated. Int J Radiat Oncol Biol Phys 2013;86:516-22.

52. Tozzi A, Comito T, Alongi F, et al. SBRT in unresectable advanced pancreatic cancer: preliminary results of a monoinstitutional experience. Radiat Oncol 2013;8:148.

53. Comito T, Cozzi L, Clerici E, et al. Can Stereotactic Body 
Radiation Therapy Be a Viable and Efficient Therapeutic Option for Unresectable Locally Advanced Pancreatic Adenocarcinoma? Results of a Phase 2 Study. Technol Cancer Res Treat 2017;16:295-301.

54. Herman JM, Chang DT, Goodman KA, et al. Phase 2 multi-institutional trial evaluating gemcitabine and stereotactic body radiotherapy for patients with locally advanced unresectable pancreatic adenocarcinoma. Cancer 2015;121:1128-37.

55. Rao AD, Sugar EA, Chang DT, et al. Patient-reported outcomes of a multicenter phase 2 study investigating gemcitabine and stereotactic body radiation therapy in locally advanced pancreatic cancer. Pract Radiat Oncol 2016;6:417-24.

56. Petrelli F, Comito T, Ghidini A, et al. Stereotactic Body Radiation Therapy for Locally Advanced Pancreatic Cancer: A Systematic Review and Pooled Analysis of 19 Trials. Int J Radiat Oncol Biol Phys 2017;97:313-22.

57. Zhong J, Patel K, Switchenko J, et al. Outcomes for patients with locally advanced pancreatic adenocarcinoma treated with stereotactic body radiation therapy versus conventionally fractionated radiation. Cancer 2017;123:3486-93.

58. Dohopolski MJ, Glaser SM, Vargo JA, et al. Stereotactic body radiotherapy for locally-advanced unresectable pancreatic cancer-patterns of care and overall survival. J Gastrointest Oncol 2017;8:766-77.

59. Crane CH. Hypofractionated ablative radiotherapy for locally advanced pancreatic cancer. J Radiat Res 2016;57 Suppl 1:i53-i7.

60. Krishnan S, Chadha AS, Suh Y, et al. Focal Radiation Therapy Dose Escalation Improves Overall Survival in Locally Advanced Pancreatic Cancer Patients Receiving Induction Chemotherapy and Consolidative Chemoradiation. Int J Radiat Oncol Biol Phys 2016;94:755-65

61. Ben-Josef E, Schipper M, Francis IR, et al. A phase I/ II trial of intensity modulated radiation (IMRT) dose escalation with concurrent fixed-dose rate gemcitabine

Cite this article as: Blakaj A, Stein SM, Khan SA, Johung KL. Review and current state of radiation therapy for locally advanced pancreatic adenocarcinoma. J Gastrointest Oncol 2018;9(6):1027-1036. doi: 10.21037/jgo.2018.03.07
(FDR-G) in patients with unresectable pancreatic cancer. Int J Radiat Oncol Biol Phys 2012;84:1166-71.

62. Wo JY, Niemierko A, Ryan DP, et al. Tolerability and Long-term Outcomes of Dose-Painted Neoadjuvant Chemoradiation to Regions of Vessel Involvement in Borderline or Locally Advanced Pancreatic Cancer. Am J Clin Oncol 2018;41:656-61.

63. Rao AD, Feng Z, Shin EJ, et al. A Novel Absorbable Radiopaque Hydrogel Spacer to Separate the Head of the Pancreas and Duodenum in Radiation Therapy for Pancreatic Cancer. Int J Radiat Oncol Biol Phys 2017;99:1111-20.

64. Brahmer JR, Tykodi SS, Chow LQ, et al. Safety and activity of anti-PD-L1 antibody in patients with advanced cancer. N Engl J Med 2012;366:2455-65.

65. Royal RE, Levy C, Turner K, et al. Phase 2 trial of single agent Ipilimumab (anti-CTLA-4) for locally advanced or metastatic pancreatic adenocarcinoma. J Immunother 2010;33:828-33.

66. Clark CE, Beatty GL, Vonderheide RH. Immunosurveillance of pancreatic adenocarcinoma: insights from genetically engineered mouse models of cancer. Cancer Lett 2009;279:1-7.

67. Kunk PR, Bauer TW, Slingluff CL, et al. From bench to bedside a comprehensive review of pancreatic cancer immunotherapy. J Immunother Cancer 2016;4:14.

68. Deng L, Liang H, Burnette B, et al. Irradiation and antiPD-L1 treatment synergistically promote antitumor immunity in mice. J Clin Invest 2014;124:687-95.

69. Vatner RE, Cooper BT, Vanpouille-Box C, et al. Combinations of immunotherapy and radiation in cancer therapy. Front Oncol 2014;4:325.

70. Le QT, Shirato H, Giaccia AJ, et al. Emerging Treatment Paradigms in Radiation Oncology. Clin Cancer Res 2015;21:3393-401.

71. Azad A, Yin Lim S, D'Costa Z, et al. PD-L1 blockade enhances response of pancreatic ductal adenocarcinoma to radiotherapy. EMBO Mol Med 2017;9:167-80. 